\title{
ATUAÇÃO DA ENFERMEIRA OBSTETRA NA COMUNIDADE ANHANGUERA, CAMPO GRANDE (MS), NA PREVENÇÃO DO CÂNCER CÉRVICO-UTERINO*
}

Sandra L. Felix de Freitas**

Sandra Lúcia Arantes***

Sonia Maria Oliveira de Barros ****

FREITAS, S.L.F.; ARANTES, S.L.; BARROS, S.M.O. Atuação da enfermeira obstetra na comunidade Anhanguera, Campo Grande (MS), na prevenção do câncer cérvico-uterino. Rev.latino-am.enfermagem, Ribeirão Preto, v. 6, n. 2, p. 57-64, abril 1998.

Este é um estudo transversal, realizado entre as mulheres da Comunidade Anhanguera, com os objetivos de: conhecer o perfil demográfico, sócio-cultural, ginecológico e obstétrico, avaliar o comportamento preventivo em saúde, e elaborar um plano de assistência de enfermagem obstétrica. O instrumento de pesquisa foi um formulário aplicado durante visitas domiciliárias a 104 mulheres. Nossos resultados mostraram que 23,08\% desconhecia o exame preventivo de câncer, 50,00\% nunca o realizou, $77,88 \%$ apresentava queixas ginecológicas mas, $62,96 \%$ não procuraram os serviços de saúde. Com base nos diagnósticos de enfermagem encontrados foi elaborado um plano de assistência de enfermagem e educação em saúde às mulheres da comunidade.

UNITERMOS: neoplasia de colo uterino, enfermagem obstétrica, prevenção \& controle, diagnóstico de enfermagem

\section{INTRODUÇÃO}

O presente trabalho é um estudo transversal sobre alguns aspectos da prevenção do câncer cérvico-uterino na comunidade Anhanguera, favela situada na Bacia do Córrego Bandeira, no Município de Campo Grande, Estado de Mato Grosso do Sul, no ano de 1995.

$\mathrm{O}$ interesse por esse tema surgiu diante das atividades desempenhadas por uma das autoras que atua como docente da Disciplina Enfermagem na Assistência à Saúde da Mulher e da Criança, no Curso de Graduação em Enfermagem e Obstetrícia da Universidade Federal de Mato Grosso do Sul.

Inicialmente as atividades das docentes na Comunidade Anhanguera eram vinculadas a um projeto de extensão que visava assistir à criança desnutrida. Durante o desenvolvimento deste projeto, as docentes da área Enfermagem Pediátrica perceberam a necessidade de intervenções de enfermagem relacionadas à saúde da mulher, especialmente no que se refere à prevenção do câncer ginecológico. Desta forma, então, iniciou-se um outro projeto de extensão, visando um programa de prevenção dirigido à todas as mulheres da comunidade.

A primeira fase deste projeto resultou no presente trabalho que visou conhecer o perfil demográfico, sóciocultural, ginecológico e obstétrico das mulheres da comunidade, a fim de subsidiar o planejamento das atividades de enfermagem.

Foram selecionados para estudo alguns aspectos referentes ao programa de prevenção do câncer cérvicouterino, tais como: fatores de risco, conhecimento das mulheres com relação ao tema, e quanto à realização e periodicidade do exame, por se constituir, ainda em nossos dias, um grave problema de Saúde Pública no Brasil e, especialmente, no Estado de Mato Grosso do Sul, tendo em vista suas altas taxas de morbidade e mortalidade, bem como a estreita relação da doença com o baixo nível socio-econômico.

Os fatores de risco associados ao câncer cérvicouterino são: dificuldade de acesso ou utilização dos serviços de saúde (MCMULLIN $\left.{ }^{13}, 1992\right)$, baixo padrão sócio-econômico, início das atividades sexuais antes dos

\footnotetext{
* Trabalho extraído da Monografia do Curso de Especialização em Enfermagem Obstétrica/1996, UNIFESP/EPM

** Professora Auxiliar da Universidade Federal de Mato Grosso do Sul. Especialista em Enfermagem Obstétrica pela UNIFESP/ EPM. COREN 37125/MS

*** Professora Assistente da Universidade Federal de Mato Grosso do Sul. Mestre em Enfermagem Obstétrica pela Escola Paulista de Medicina. COREN 22691/MS

**** Professora Adjunto da Universidade Federal de São Paulo/EPM. Doutora em Enfermagem Materna e Infantil pela UNIFESP/ EPM. COREN 14337/SP
} 
20 anos, multiparidade (SILVEIRA ${ }^{22}, 1989$; HERRERO $^{9}$ et al., 1990; MCMULLIN ${ }^{13}$, 1992; PIOLI $^{17}$ et al., 1993; GIUSA $^{7}$ et al., 1994), freqüência da atividade sexual aumentada (BENRUBI $\left.{ }^{4}, 1986\right)$, primiparidade precoce, promiscuidade sexual da mulher ou do parceiro, doenças sexualmente transmissíveis, especialmente as causadas pelos vírus HPV e herpes simples tipo II (BARBOSA ${ }^{3}$ et al., 1991; MCMULLIN ${ }^{13}$, 1992), relações sexuais com homens carcinogênicos (BENRUBI ${ }^{4}, 1986$; TERREIRO $^{23}$, 1987; ALVES $^{1}$ et al., 1992), higiene sexual inadequada (BARBOSA ${ }^{3}$ et al., 1991; PIOLI $^{17}$ et al., 1993) e o tabagismo (HERRERO ${ }^{9}$ et al., 1990; MCMULLIN $^{13}$, 1992; ALVES $^{1}$ et al., 1992).

Prevenir é se antecipar ao acontecimento, impedindo que um fato ocorra ou mesmo que tenha continuidade, portanto a atitude preventiva ao câncer cérvico-uterino deve ser abrangente, a fim de evitar o processo de cancerização ou mesmo a interrupção da evolução de uma lesão pré-maligna, para isso utilizandose de todos os recursos diagnósticos disponíveis (SILVEIRA $^{22}$, 1989).

A prevenção do câncer cérvico-uterino se baseia essencialmente no rastreamento (screening) na população, no diagnóstico preciso do grau da lesão e no tratamento adequado, sendo que a população a ser rastreada constitui-se em todas as mulheres que apresentam probabilidade de ter lesões pré-cancerosas detectáveis pelo exame citológico (REIS ${ }^{18}$ et al., 1992).

No entanto, para que uma alta porcentagem da população seja rastreada e desta forma realmente beneficiada pelo programa de prevenção ao câncer cérvico-uterino é fundamental que os serviços de saúde estejam equipados e organizados para realizar o exame com regularidade e que as mulheres, por sua vez, manifestem o comportamento preventivo em saúde, buscando estes serviços.

Segundo KATALSKI ${ }^{10}$ (1977), o comportamento preventivo em saúde é qualquer atitude tomada por uma pessoa que se acredita saudável, com o propósito de prevenir doenças ou detectá-las num estado assintomático.

Para que ocorra o comportamento preventivo em saúde, ou seja a adoção de uma determinada prática de saúde, é necessária a aquisição anterior, de um conhecimento cientificamente correto, a fim de que o indivíduo possa, após uma avaliação da situação, decidirse quanto a adequação da prática (CANDEIAS \& MARCONDES $^{6}, 1979$ ). Para tanto, faz-se necessário um programa de educação em saúde bastante amplo, e que contemple os aspectos de prevenção de doenças e de preservação da saúde.

"A educação pouco voltada para a profilaxia determina grandes danos, sobretudo em certas patologias em que é vital o diagnóstico precoce”, portanto, considera-se que, a educação em saúde constitui o processo mais eficiente das ações preventivas, pois é um instrumento de transformação social que visa mudanças de comportamento e reformulação de hábitos. (REZENDE $\left.{ }^{19}, 1989\right)$.

A enfermeira obstetra pode e deve estar envolvida nesse processo de mudança de comportamento, atuando diretamente com a mulher, família e comunidade em que a mesma se encontra inserida, com vistas a obtenção e manutenção da saúde.

Segundo MARCON ${ }^{11}$ (1990), um dos objetivos da enfermeira é encontrar meios que favoreçam a motivação e a adoção de medidas de promoção da saúde e de prevenção de doenças, tanto a nível individual como coletivo.

Esta profissional tem suas ações voltadas, tanto para a assistência às clientes como para os aspectos preventivos, sendo que nestes, sua atuação está intimamente ligada à educação em saúde. No entanto, para que as ações de enfermagem possam ser desenvolvidas de forma adequada e eficiente faz-se necessário, inicialmente, realizar o diagnóstico de enfermagem, a fim de tornar possível a elaboração de um plano de atuação.

Segundo SHOEMAKER ${ }^{21}$ (1985), diagnóstico de enfermagem é uma avaliação clínica do indivíduo, família ou da comunidade, realizada através da coleta e análise dos dados, e fornece a base para as prescrições de enfermagem, pelas quais o enfermeiro é responsável.

GORDON $^{8}$ (1987) definiu o diagnóstico de enfermagem como sendo atividade realizada pelo enfermeiro, para descrever problemas de saúde presentes ou potenciais, os quais o enfermeiro foi capacitado e licenciado para tratar.

$\mathrm{Na}$ definição da North American Nursing Diagnosis Association - NANDA ${ }^{14}$ (1990) "diagnóstico de enfermagem é o julgamento clínico das respostas do indivíduo, da família ou da comunidade aos processos vitais ou aos problemas de saúde atuais ou potenciais, os quais fornecem a base para a seleção das intervenções de enfermagem, para atingir resultados, pelos quais a enfermeira é responsável".

\section{OBJETIVOS}

- conhecer o perfil demográfico, sócio-cultural, ginecológico e obstétrico das mulheres da comunidade Anhanguera;

- avaliar o conhecimento das mulheres quanto ao programa de prevenção do câncer cérvico-uterino;

- identificar as queixas ginecológicas atuais, bem como o comportamento das mulheres frente às mesmas;

- elaborar um plano de educação em saúde e assistência 
de enfermagem para a comunidade, baseado nos diagnósticos de enfermagem, segundo a taxonomia da NANDA (North American Nursing Diagnosis Association) mais freqüentemente encontrados.

\section{MATERIAL E MÉTODO}

A população de estudo foi constituída por $73,24 \%$ das 142 mulheres pertencentes à Comunidade Anhanguera, ou seja, 104 mulheres.

$\mathrm{O}$ instrumento de pesquisa utilizado foi um formulário semi-estruturado, constituído por questões abertas e fechadas. Foi realizado um pré-teste do instrumento em 21 mulheres da comunidade para os ajustes necessários.

Todas as mulheres foram entrevistadas em suas residências após o consentimento pós-informado em participar da pesquisa.

As visitas à comunidade foram realizadas todas as segundas-feiras, no mês de maio de 1995. Para que se pudesse atingir toda a população de mulheres, foram realizadas, também, visitas nos finais de semana, uma vez que muitas trabalhavam fora. Ainda assim 17 mulheres $(11,97 \%)$ da comunidade não puderam ser entrevistadas.

Participaram também das visitas domiciliares e coleta de dados todos os alunos da $2^{\mathrm{a}}$ série do Curso de Graduação em Enfermagem e Obstetrícia da Universidade Federal de Mato Grosso do Sul, após treinamento realizado por uma das autoras.

As variáveis de estudo foram:

\section{I- Variáveis demográfico-sócio-culturais:}

- Idade em anos completos

- Escolaridade.

- Situação Marital - que foi categorizada em presença ou ausência de companheiro fixo por ocasião da pesquisa.

\section{II- Variáveis Obstétricas:}

- Número de gestações

- Número de Abortamentos

- Paridade

- Tipos de Parto

- Prática do aleitamento materno

III- Variáveis Ginecológicas:

- Conhecimento sobre o exame preventivo de câncer de colo de útero

- Realização e regularidade do exame preventivo

- Existência de queixas ginecológicas e procura de assistência médica para resolvê-las.
IV-Diagnósticos de Enfermagem de acordo a taxonomia da NANDA, segundo sua(s) característica(s) definidora(s).

\section{RESULTADOS E DISCUSSÃO}

A análise dos resultados mostrou que a população estudada expressa uma realidade conhecida no Estado de Mato Grosso do Sul.

Apesar da comprovada eficácia da tecnologia utilizada para o diagnóstico e a detecção da displasia (lesão precursora do câncer cérvico-uterino), bem como de carcinomas invasores e de um grande número de agentes infecciosos, baseados em informações recebidas do Núcleo de Estatística Vital, SÃO PAULO. S.E.S. ${ }^{20}$ (1995), observamos que, nos últimos 10 anos vem ocorrendo um constante aumento no número de óbitos pelo câncer cérvico-uterino, especialmente nas mulheres com 50 anos ou mais.

Essa alta mortalidade pode ser um indicativo de falhas no diagnóstico precoce da displasia, que podem ser decorrentes à vários fatores, tais como: dificuldade de acesso ao programa de prevenção ao câncer cérvicouterino em decorrência da não implantação do mesmo em todas as unidades básicas de saúde, a não utilização destes serviços por parte da população de risco e falta de uma adequada educação em saúde às mulheres.

Tabela 1 - Distribuição das mulheres da Comunidade Anhanguera segundo as variáveis demográfico-sócioculturais selecionadas

\begin{tabular}{ccc}
\hline IDADE (ANOS) & N & \% \\
\hline$<20$ & 15 & 14,42 \\
$20 \mid-30$ & 35 & 33,65 \\
$30 \mid-40$ & 19 & 18,27 \\
$40 \mid-50$ & 17 & 16,35 \\
50 ou mais & 18 & 17,31 \\
TOTAL & 104 & 100,00 \\
\hline ESCOLARIDADE & $\mathbf{N}$ & $\mathbf{\%}$ \\
\hline nenhuma & 30 & 28,85 \\
I grau incompleto & 67 & 64,42 \\
II grau incompleto & 7 & 6,73 \\
TOTAL & 104 & 100,00 \\
\hline SITUAÇ̃OMARITAL & $\mathbf{N}$ & $\mathbf{\%}$ \\
\hline com companheiro fixo & 71 & 68,27 \\
sem companheiro fixo & 33 & 31,73 \\
TOTAL & 104 & 100,00 \\
\hline
\end{tabular}


Quanto às variáveis demográfico-sócio-culturais (Tabela 1) nossos resultados mostraram que a maioria das mulheres entrevistadas encontrava-se na idade reprodutiva, implicando em necessidade de abordar questões como: planejamento familiar, importância do pré-natal, vantagens do parto normal e do aleitamento materno, cuidados de higiene pessoal e a prevenção do câncer ginecológico.

Optamos por priorizar as atividades relacionadas à prevenção do câncer cérvico-uterino por ser este o câncer de maior incidência entre as mulheres no Brasil (NISIDA \& PINOTTI ${ }^{15}, 1992$ ). Segundo o Ministério da Saúde (BRASIL ${ }^{5}$, 1986) dentre as neoplasias malignas, no Brasil, o câncer cérvico-uterino e de mama constituem as principais causas de óbito na população feminina de 15 anos ou mais, sendo que o câncer cérvico-uterino e de mama somam juntos, aproximadamente $25,00 \%$ do total destes óbitos (ZEFERINO ${ }^{26}$ et al., 1987).

Um dos motivos de maior preocupação com o câncer cérvico-uterino é o fato dele atingir sobretudo mulheres num período em que a maioria delas já não procura o ginecologista regularmente, uma vez que já não se preocupa tanto com a reprodução, fato este que dificulta o diagnóstico precoce da patologia $\left(\mathrm{PIOLI}^{17}\right.$ et al., 1993).

Um estudo retrospectivo realizado entre as pacientes com câncer cérvico-uterino atendidas no Hospital das Clínicas da Universidade Federal de Uberlândia (HCUFU), no período de 1984 a 1988, mostrou que o carcinoma cérvico-uterino ocorreu com maior freqüência na faixa etária entre 35 e 60 anos (PIOLI ${ }^{17}$ et al., 1993).

Neste estudo, $28,85 \%$ das mulheres entrevistadas, referia nenhuma escolaridade, e $64,42 \%$ tinha apenas o primeiro grau incompleto, determinando que as atividades educativas de enfermagem requeriam abordagem simples e direta, a fim de serem captadas e entendidas por todas as mulheres.

Com relação às variáveis obstétricas (Tabela 2) chamou-nos a atenção o fato de que $48,08 \%$ das mulheres entrevistadas já tinham tido 4 (quatro) gestações ou mais, $25,96 \%$ pelo menos 1 (um) abortamento anterior, $38,46 \%$ tiveram 4 (quatro) partos ou mais, tendo em vista que a multiparidade (SILVEIRA ${ }^{22}$, 1989; BARBOSA ${ }^{3}$ et al., 1991; GIUSA $^{7}$ et al., 1994) bem como o número de abortamento são fatores de risco relevantes para o câncer cérvico-uterino (UCHIMURA ${ }^{24}, 1993$ ).

No que se refere à situação marital, a maioria $(68,27 \%)$ referiu ter um companheiro fixo por ocasião da pesquisa.
Tabela 2 - Distribuição das mulheres da Comunidade Anhanguera, segundo as variáveis obstétricas

\begin{tabular}{ccc}
\hline NÚMERO DE GESTACÕES & N & \% \\
\hline 0 & 5 & 4,80 \\
1 & 14 & 13,46 \\
2 & 11 & 10,58 \\
3 & 24 & 23,08 \\
4 ou mais & 50 & 48,08 \\
TOTAL & 104 & 100,00 \\
\hline ABORT OS ANTERIORES & $\mathbf{N}$ & $\mathbf{\%}$ \\
\hline 0 & 77 & 74,04 \\
1 & 13 & 12,50 \\
2 & 8 & 7,69 \\
3 ou mais & 6 & 5,77 \\
TOTAL & 104 & 100,00 \\
\hline PARIDADE & $\mathbf{N}$ & $\mathbf{\%}$ \\
\hline 0 & 7 & 6,73 \\
1 & 15 & 14,42 \\
2 & 17 & 16,35 \\
3 & 25 & 24,04 \\
4 ou mais & 40 & 38,46 \\
TOTAL & 104 & 100,00 \\
\hline TIPOS DE PARTO & $\mathbf{N}$ & $\mathbf{\%}$ \\
\hline normal & 317 & 79,25 \\
cesárea & 83 & 20,75 \\
TOTAL & 400 & 100,00 \\
\hline
\end{tabular}

Uma pesquisa realizada nos ambulatórios de ginecologia do Hospital Universitário da Universidade Federal de Santa Maria demonstrou que 39,1\% das alterações nos exames citopatológicos e/ou colposcópicos ocorreram nas multíparas (BARBOSA ${ }^{3}$ et al., 1991).

Ainda na pesquisa realizada no HCUFU, PIOLI $^{17}$ et al. (1993) referiram que a ocorrência da doença foi maior $(57,2 \%)$ em mulheres com cinco filhos ou mais.

Quanto aos tipos de parto, nesta pesquisa observou-se que, dos 400 partos ocorridos, 79,25\% foram normais. Apesar de todas as vantagens do parto normal não podemos deixar de observar que a má assistência obstétrica a que está exposta a nossa população mais carente, é a responsável por muitas das alterações morfológicas cervicais (TERREIRO ${ }^{23}, 1987$ ).

Sem uma assistência obstétrica adequada, o colo uterino poderá ficar com seqüelas que muito contribuirão para que se desenvolvam processos inflamatórios e neoplásicos. As lesões não corrigidas do colo tornam a mucosa endocervical exposta ao meio vaginal, e, consequentemente, ao seu pH ácido, às agressões de agentes infecciosos (fungos, vírus e bactérias) e à possível oncogenicidade do esmegma e do esperma (TERREIRO $^{23}, 1987$ ).

Para VIEIRA ${ }^{25}$ (1987) um dos aspectos importantes da prevenção das lesões malignas do colo uterino é evitar e corrigir todas as lacerações cervicais, principalmente as obstétricas.

No que se refere à pratica do aleitamento materno, 
94,66\% das crianças nascidas vivas foram amamentadas. Destas, 73,39\% por 6 meses ou mais. Apenas 28 crianças,

7 delas natimortos, não foram amamentadas.

$\mathrm{Na}$ Tabela 3 podemos observar que, apesar da maioria das mulheres entrevistadas ter declarado algum conhecimento sobre o exame preventivo do câncer cérvico-uterino, apenas 50,0\% delas já o haviam realizado. Cerca de $23,08 \%$ desconheciam totalmente este exame.

O exame preventivo, ou Papanicolau (coleta tríplice cervicovaginal) é um dos exames utilizados no diagnóstico do câncer cérvico-uterino, sendo os demais: a colposcopia, a biópsia dirigida e estudo anatomopatológico (ALVES ${ }^{1}$ et al., 1992).

Nos países subdesenvolvidos o câncer cérvicouterino continua sendo a doença ginecológica mais importante (MARTINS ${ }^{12}$ et al., 1992), e isto se deve, principalmente, ao baixo percentual de mulheres que se submetem ao rastreamento pelo exame preventivo (GIUSA ${ }^{7}$ et al., 1994).

Tabela 3 - Distribuição das mulheres da Comunidade Anhanguera, segundo as variáveis ginecológicas

\begin{tabular}{|c|c|c|}
\hline $\begin{array}{l}\text { CONHECIMENTO SOBRE O } \\
\text { EXAME PREVENTIVO }\end{array}$ & $\mathrm{N}$ & $\%$ \\
\hline $\operatorname{Sim}$ & 80 & 76,92 \\
\hline Não & 24 & 23,08 \\
\hline TOTAL & 104 & 100,00 \\
\hline REALIZAÇAOO DO EXAME & $\mathrm{N}$ & $\%$ \\
\hline Não & 52 & 50,00 \\
\hline $\operatorname{Sim}$ & 52 & 50,00 \\
\hline TOTAL & 104 & 100,00 \\
\hline $\begin{array}{l}\text { INTERVALO DA REALIZAÇÃO } \\
\text { (meses) }\end{array}$ & $\mathrm{N}$ & $\%$ \\
\hline $0 \mid-12$ & 24 & 46,15 \\
\hline $12 \mid-24$ & 11 & 21,15 \\
\hline 24 ou mais & 17 & 32,70 \\
\hline TOTAL & 52 & 100,00 \\
\hline $\begin{array}{c}\text { RETORNOAO MÉDICO APÓS } \\
\text { OPREVENTIVO }\end{array}$ & $\mathbf{N}$ & $\%$ \\
\hline $\operatorname{Sim}$ & 36 & 69,23 \\
\hline Não & 16 & 30,77 \\
\hline TOTAL & 52 & 100,00 \\
\hline $\begin{array}{l}\text { QUEIXAS GINECOLÓGICAS } \\
\text { ATUAIS }\end{array}$ & $\mathbf{N}$ & $\%$ \\
\hline $\operatorname{Sim}$ & 81 & 77,88 \\
\hline Não & 23 & 22,12 \\
\hline TOTAL & 104 & 100,00 \\
\hline $\begin{array}{c}\text { MULHERES COM QUEIXAS } \\
\text { QUE PROCURARAM } \\
\text { ASSISTENCIAMÉDICA } \\
\end{array}$ & $\mathrm{N}$ & $\%$ \\
\hline $\operatorname{Sim}$ & 30 & 37,04 \\
\hline Não & 51 & 62,96 \\
\hline TOTAL & 81 & 100,00 \\
\hline
\end{tabular}

Quanto ao intervalo da realização do exame, no presente estudo, observou-se que apenas $46,15 \%$ o faziam com intervalo menor que doze meses. $\mathrm{O}$ intervalo recomendado pelo Ministério da Saúde (BRASIL $\left.{ }^{5}, 1986\right)$ para as mulheres cujo resultado do preventivo foi negativo para células neoplásicas é de um ano, sendo que, para as clientes que o fazem regularmente (todos os anos) e obtiverem duas ou mais citologias negativas, o intervalo entre uma coleta e outra pode ser ampliado para dois ou três anos.

Segundo BENRUBI ${ }^{4}$ (1986), a American Cancer Society recomenda que as mulheres com risco de câncer cérvico-uterino devem fazer o exame anualmente, sendo que um dos fatores de risco foi definido como mais de dois parceiros sexuais durante toda a vida.

Para ZEFERINO ${ }^{26}$ et al. (1987) entre uma mulher que já fez o preventivo do câncer, ainda que há mais de um ano, e outra que nunca o realizou, deve-se priorizar a segunda, pois nesta o risco de se encontrar o câncer cérvico-uterino é maior.

A pesquisa constatou que, entre as mulheres que já haviam feito o exame preventivo, $30,77 \%$ não haviam retornado ao serviço de saúde após o mesmo, o que invalida essa prática de saúde, uma vez que pode ter havido a detecção da lesão precursora do carcinoma invasivo através do resultado do exame, mas não foi possível o tratamento da mesma.

Sabemos que o principal objetivo dos programas de prevenção do câncer cérvico-uterino é prevenir o carcinoma invasivo através da detecção, diagnóstico e tratamento precoce da patologia quando a cura ainda pode ser alcançada (ORGANIZAÇÃO PAN-AMERICANA DE SAÚDE $\left.{ }^{16}, 1985\right)$.

A neoplasia intra-epitelial cervical, forma precursora do carcinoma invasivo de colo uterino, é curável em praticamente $100 \%$ dos casos, e com um baixo custo. No entanto, as chances de tratamento e cura na forma invasora da doença são muito menores (ZEFERINO $^{26}$ et al., 1987), por isso é indispensável o retorno ao médico após a realização do preventivo para que seja feito um tratamento adequado à situação detectada.

Quanto à presença de queixas ginecológicas atuais, $77,88 \%$ das mulheres da comunidade as referiram, sendo que a maioria delas tinha mais de uma queixa. Das 104 mulheres entrevistadas $39,42 \%$ referiram corrimento vaginal, $15,38 \%$ referiram prurido vulvar, $37,50 \%$ presença de dor no baixo ventre, $39,42 \%$ ocorrência de dismenorréia, $31,73 \%$ de irregularidades no ciclo menstrual e 40,38\% referiram alterações na quantidade do fluxo menstrual.

As mulheres com câncer cérvico-uterino, podem apresentar alguns sinais e sintomas, principalmente as que se encontram em um estágio mais avançado da 
doença, sendo que os mais comuns são: sangramento vaginal especialmente após as relações sexuais ou a ducha vaginal, corrimento vaginal fétido, dor em baixo ventre, fraqueza, anorexia e emagrecimento (BENRUBI ${ }^{4}, 1983$; MCMULLIN $^{13}$, 1992; PIOLI $^{17}$ et al., 1993).

Sabemos que todos os fatores de risco para o câncer cérvico-uterino estão voltados para as agressões do epitélio cervical, entre elas as infecções genitais, por esse motivo é fundamental o tratamento adequado e precoce das mesmas (SILVEIRA $\left.{ }^{22}, 1989\right)$.

No entanto, observou-se neste estudo que, das 81 mulheres que referiam queixas ginecológicas, apenas $37,04 \%$ buscaram assistência médica para o tratamento das mesmas, fato que nos mostrou mais uma vez, a necessidade da atuação da enfermeira obstetra nesta comunidade, tanto no desenvolvimento de atividades assistenciais de enfermagem como nas de educação em saúde.

Segundo ARAÚJO² (1992), a educação em saúde é a responsável pelo constante declínio, nos últimos anos, da taxa de mortalidade pelo câncer cérvico-uterino nos
Estados Unidos, pois mais mulheres estão sendo orientadas a fazer um exame anual que inclui o esfregaço de Papanicolau.

Os diagnósticos de enfermagem (Tabela 4) subsidiaram a elaboração de um plano de assistência de enfermagem obstétrica, com a participação inclusive de outras docentes da Disciplina Enfermagem na Assistência à Saúde da Mulher e da Criança e também, dos alunos do $2^{\circ}$ ano do Curso de Graduação em Enfermagem.

Após a elaboração, o plano de assistência de enfermagem obstétrica foi apresentado aos representantes da comunidade Anhanguera, para que os mesmos tomassem conhecimento das ações propostas. Este momento foi muito importante para a interação das enfermeiras obstetras com a comunidade, uma vez que os seus representantes puderam até mesmo, solicitar a inclusão de intervenções adicionais visando uma participação mais ativa das mulheres nas unidades de saúde por elas utilizadas.

O plano foi constituído por atividades de enfermagem educativas e assistenciais.

Tabela 4 - Diagnósticos de enfermagem (NANDA) encontrados nas mulheres da Comunidade Anhanguera

DIAGNÓSTICO $\quad$ CARACTERISTICA(S) DEFINIDORA(S)

1.2.1.1 - Potencial para infecção

Defesa inadequada por mudança no $\mathrm{pH}$ das secreções (corrimento vaginal)

1.6.2.1.2.1 - Integridade da pele prejudic ada

1.6.2.1.2.2 - Potencial paraprejúzo na integridade da pele

5.4 - Comportamento para elevar nivel de saúde

6.4.2 - Alteração na manutenção da Falta demonstrada de conhecimento sobre práticas básicas de saúde saúde

Ruptura da superficie da pele e destruição das camadas de pele (pelo prurido vulvar)

Presença de fatores de risco como: secreções e umidade (corrimento vaginal)

Desejo observado e/ou relatado de aumentar práticas de controle da saúde (mulheres fazem o preventivo do câncer cérvico-uterino com intervalo menor que 12 meses)

(as que nunca ouviram falar no preventivo do câncer cérvico-uterino) História de falta de comportamento em busca de saúde:

- As que nunca realizaram o preventivo

- As que o fazem com intervalo de 12 |- 24 meses

- As que fazem com intervalo igual ou maior que 24 meses

- Mulheres com queixas ginecológicas que não procuram assistência médica

8.1.1 - Déficit de conhecimento Verbalização do problema: sobre a prevenção do câncer - Mulheres que nunca ouviram falar da prevenção do câncer cérvico-uterino

9.1.1 - Dor

\section{ginec ológico}

Comunicação de expressão de dor:

- Mulheres que referiram dor no baixo ventre

- Mulheres que referiram dismenorréia
$\%$

39,42

15,38

39,42

23,08

23,08

50,00

21,15

32,70

62,96

23,08

37,50

39,42

\section{CONCLUSÕES}

Nossos resultados permitiram-nos chegar às seguintes conclusões:
- A maioria das mulheres da comunidade encontrava-se no período reprodutivo, com nenhuma ou pouca escolaridade, e com companheiro fixo.

- Com relação às variáveis obstétricas, destaca-se o alto 
percentual de multíparas $(38,46 \%), 79,25 \%$ de partos normais e $20,75 \%$ de cesáreas.

Quanto à pratica do aleitamento materno $94,66 \%$ das crianças nascidas vivas foram amamentadas sendo que, destas, $73,39 \%$ o foram por 6 meses ou mais.

- No que se refere ao conhecimento do programa de prevenção do câncer cérvico-uterino, cerca de 23,08\% das mulheres entrevistadas referiram desconhecê-lo, e apenas, $50,00 \%$ referiram já ter feito o exame preventivo pelo menos uma vez, destas, $53,85 \%$ o fizeram com intervalo superior a 12 meses, e 30,77 \% não retornaram ao serviço de saúde para conhecimento do resultado e posterior tratamento, se necessário.

Quanto as mulheres que apresentaram queixas ginecológicas $(77,88 \%)$ apenas $37,04 \%$ procuraram assistência médica para resolvê-las.

As queixas referidas, por ordem de ocorrência, foram: alterações na quantidade do fluxo menstrual $(40,38 \%)$, corrimento vaginal $(39,42 \%)$, dismenorréia $(39,42 \%)$, dor no baixo ventre $(37,50 \%)$, irregularidades no ciclo menstrual $(31,73 \%)$ e prurido vulvar $(15,38 \%)$.

\section{CONSIDERAÇÕES FINAIS}

O planejamento das atividades educativas contemplou os seguintes aspectos:

- Anatomia e fisiologia feminina

- Higiene Feminina

- Doenças sexualmente transmissíveis

- Planejamento Familiar

- Auto-exame de mamas

- Exame preventivo do Câncer cérvico-uterino: quando, como e porque.

Como estratégias de aprendizagem foram propostas palestras para pequenos grupos e em diferentes horários, discussões em grupo, orientações individuais domiciliares e apresentações teatrais.

As principais atividades assistenciais de enfermagem propostas à comunidade foram:

- Agendamento e realização do exame preventivo pelas enfermeiras obstetras, assim como retorno para conhecimento do resultado;

- Consulta de enfermagem no centro de saúde e domiciliária, se necessário;

- Visita domiciliar a todas as mulheres que realizaram o exame preventivo;

- Encaminhamento às especialidades médicas e ao serviço odontológico, quando indicado.

\section{ROLE OF THE OBSTETRIC NURSE IN THE ANHANGUERA COMMUNITY, CAMPO GRANDE (MS), FOR THE PREVENTION OF CERVICAL UTERINE CANCER}

This is a transversal study, developed between women of the Anhanguera community, with the objectives of knowing the demographic, sociocultural, gynecological and obstetric profile and of assessing health preventive behaviors in order to elaborate a plan of obstetric nursing assistance. The research tool was a form applied at home during visits to 104 women. Our results showed that 23,08\% did not know about cancer prevention, 50,00\% never performed it, 77,88\% presented gynecological complaints but, $62,96 \%$ of these never looked for health services. Based on nursing diagnoses a plan of nursing care and health education was elaborated for the women from this community.

KEY WORDS: neoplasia of uterine cervix, obstetric nursing, prevention \& control, nursing diagnosis

\section{ACTUACIÓN DE LA ENFERMERA OBSTÉTRA EN LA COMUNIDAD ANHANGUERA, CAMPO GRANDE (MS), EN LA PREVENCIÓN DEL CÁNCER DE CUELLO UTERINO}

Este es un estudio transversal, realizado entre las mujeres de la comunidad Anhanguera, con los objetivos de conocer el perfil demográfico, el socio-económico, ginecológico y obstétrico asi como evaluar el comportamiento preventivo en salud, para la elaboración de un plan de asistencia de la enfermería obstétrica. El instrumento de esta investigación fue un cuestionario realizado en consultas a domicilio hechas a 104 mujeres. Nuestros resultados mostraron que un 23,08\% de ellas desconocía la existencia de un exame de prevención del cáncer, 50,00\% nunca lo había hecho, 77,88\% presentaba quejas ginecológicas pero, de estas, $62,96 \%$ no buscaron los servicios de salud. Basandonos en estos diagnósticos, elaboramos un plan de asistencia de enfermería y educación en salud a las mujeres de la comunidad.

TÉRMINOS CLAVES: neoplasia de cuello uterino, enfermería obstétrica, prevención y control, diagnóstico por enfermería 
REFERÊNCIAS BIBLIOGRÁFICAS

01. ALVES, A .C.; MARTINS, N.V.; DORES, G.B. das; SIMÕES, P.M.; SOUZA, R.C.; LIMA, G.R. Neoplasias malignas do colo uterino. In: LIMA, G.R de; MARTINS, N.V. Oncoginecologia. Rio de Janeiro: Atheneu, 1992. p. 105-121.

02. ARAÚJO, C.L.F. Consulta de enfermagem ginecológica: eficácia do tratamento de rotina nas vulvovaginites. Rev. Bras. Enfermagem, v.45, n.2/3, p.116-21, 1992.

03. BARBOSA, L.C.R.; RIESGO, I.S.; QUINTANA, S.M.; MARTELLY, J.U.; GAUZA, J.E.; MELLO, A.B.B.; BINATO, A.; CECHELLA, M.S.;SANTOS, R.P. Relação citologia oncótica, colposcopia e histopatologia no diagnóstico do carcinoma cérvico-uterino. J. Bras. Ginecol., v.101, n.8, p. 351-53, 1991.

04. BENRUBI, G. Doenças malignas do colo do útero. In: ELLIS, J.W.; BECKMANN, C.R.B. Manual de ginecologia. Rio de Janeiro: Prentice-Hall do Brasil, 1986.

05. BRASIL. Ministério da Saúde. Controle do câncer cérvico-uterino e de mama. Normas e manuais técnicos. Brasília: Centro de documentação do Ministério da Saúde, 1986, p. 8.

06. CANDEIAS, N.M.; MARCONDES, R.S. Diagnóstico em educação em saúde: um modelo para analisar as relações entre atitudes e práticas na área de saúde pública. Rev. Saúde Pública, São Paulo, v.13, n. 2, p.63-68, 1979.

07. GIUSA, M.G.; GONÇALVES, W.J.; BORTOLETTO, C.C.R.; SARTORI, M.G.F.; KOBATA, S.A.; BARACAT, E.C.; LIMA, G.R. Aspectos atuais no tratamento do câncer de colo uterino. Compacta Ginecol., n. ${ }^{\circ}$ 4, p.9-10, 1994.

08. GORDON, M. Nursing diagnosis: process and application. 2 ed. New York: McGraw-Hill, 1987.

09. HERRERO, R.; BRINTON, L.A.; REEVES, W.C.; BRENES, M.M.; TENORIO, F.; BRINTON, R.C. de; GAITAN, E.; GARCIA, M.; RAWLS, W.E. Sexual behavior, venereal diseases, hygiene practices, and invasive cervical cancer in a highrisk population. Cancer, v.65, n.2, p.380-85, 1990.

10. KATALSKY, M.E. The health belief model as conceptual fromwork for explaining contraceptive compliance. Health Educ. Monogr., v.5, n.3, p. 232-243, 1977.

11. MARCON, S.S. Comportamento preventivo em saúde: exploração do conceito. Rev. Gaúch. Enfermagem, Porto Alegre, v.1, n.2, p.28-34, 1990.

12. MARTINS, N.V.; FOCCHI, J.;LIMA G.R. Câncer invasor do colo uterino. In: RAMOS, J.; VALLE, J.R.; PRADO, F.C. Atualização terapêutica. São Paulo: Artes Médicas, 1992. p. 780-798.
13. MCMULLIN, M. Holistic care of the patient with cervical cancer. Nurs. Clin. North Am., v.27, n.4, p.847-57, 1992.

14. NANDA. Taxonomy I Revised with official diagnostic categories. St . Louis, 1989.

15. NISIDA, A.C.T.; PINOTTI, J.A. Programas de controle de câncer ginecológico. Rev. Ginecol. Obstet., São Paulo, v.3, n.3, p.103-104, 1992.

16. ORGANIZAÇÃO PAN-AMERICANA DE SAÚDE. Epidemiologia do câncer cérvico-uterino. Manual de normas e procedimentos para o controle do câncer cérvico-uterino. Washington, D.C., 1985.

17. PIOLI, E.R.; OLIVEIRA, N.M. de ; REZENDE, A.G. de. Caracterização de demanda de pacientes com carcinoma de colo uterino no Hospital das Clínicas da Universidade Federal de Uberlândia, Minas Gerais, Brasil, 1984-1988. Cad. Saude Pública, v.9, n.4, p.421-427, 1993.

18. REIS, A.F.F., COSTA, M.C.E.; ALMEIDA, N.C. Prevenção do câncer cérvico-uterino - princípios epidemiológicos e avaliação dos programas de screening. J. Bras. Ginecol., v.102, n. 11/12, p.445-47, 1992.

19. REZENDE, A.L.M. Saúde: dialética do pensar e do fazer. São Paulo: Cortez, 1989.

20. SÃO PAULO (Estado). Secretaria de Estado de Saúde. Dados estatísticos de mortalidade feminina por câncer no estado de Mato Grosso do Sul, 1984 a 1994. 1995, 120p. (Mimeografado).

21. SHOEMAKER, J.K. Characteristics of a nursing diagnosis. Occup. Health Nurs., v.33, p.387-89, 1985.

22. SILVEIRA, G.P.G. Sobre a prevenção do câncer ginecológico e mamário. Rev. Med. PUCRS, Porto Alegre, v.1, n.2, p.69-72, 1989.

23. TERREIRO, L.M. Câncer do colo uterino: conceito, importância, incidência e fatores de risco. In: HALBE, H.W. Tratado de ginecologia. São Paulo: Roca, 1987. v.2, p. 1494 - 504.

24. UCHIMURA, N.S. Estudo clínico, colposcópico e histopatológico da cérvice uterina de mulheres nas áreas urbana e rural da região de Maringá. São Paulo, 1993. 69p. Dissertação (Mestrado) Escola Paulista de Medicina.

25. VIEIRA, L.S. Câncer do colo uterino - prevenção e diagnóstico precoce. In: HALBE, H.W. Tratado de ginecologia. São Paulo: Roca, 1987. v.2, p. 1544- 47.

26. ZEFERINO, L.C.; PINOTTI, J.A.; TEIXEIRA, L.C. O problema do câncer ginecológico e mamário. Femina, v.15, n.3, p.198 - 212, 1987. 